\title{
Color Based Stool Region Detection in Colonoscopy Videos for Quality Measurements
}

\author{
Jayantha Muthukudage ${ }^{1}$, JungHwan $\mathrm{Oh}^{1}$, \\ Wallapak Tavanapong ${ }^{2}$, Johnny Wong ${ }^{2}$, and Piet C. de Groen ${ }^{3}$ \\ ${ }^{1}$ Department of Computer Science and Engineering, \\ University of North Texas, Denton, TX 76203 \\ ${ }^{2}$ Computer Science Department, Iowa State University, Ames, IA 50011 \\ ${ }^{3}$ Mayo Clinic College of Medicine, Rochester, MN 55905 \\ MuthukudageKumara@my. unt.edu, Junghwan. Oh@unt.edu, \\ \{tavanapo, wong\} @cs. iastate.edu
}

\begin{abstract}
Colonoscopy is the accepted screening method for detecting colorectal cancer or colorectal polyps. One of the main factors affecting the diagnostic accuracy of colonoscopy is the quality of bowel preparation. Despite a large body of published data on methods that could optimize cleansing, a substantial level of inadequate cleansing occurs in $10 \%$ to $75 \%$ of patients in randomized controlled trials. In this paper, we propose a novel approach that automatically determines percentages of stool areas in images of digitized colonoscopy video files, and automatically computes an estimate of the BBPS (Boston Bowel Preparation Scale) score based on the percentages of stool areas. It involves the classification of image pixels based on their color features using a new method of planes on RGB (Red, Green and Blue) color space. Our experiments show that the proposed stool classification method is sound and very suitable for colonoscopy video analysis where variation of color features is considerably high.
\end{abstract}

Keywords: Image Classification, Region of Interest Detection, Colonoscopy, Medical Image Analysis.

\section{Introduction}

Advances in video technology are being incorporated into today's healthcare practices. Various types of endoscopes are used for colonoscopy, upper gastrointestinal endoscopy, enteroscopy, bronchoscopy, cystoscopy, laparoscopy, wireless capsule endoscopy, and some minimally invasive surgeries (i.e., video endoscopic neurosurgery). These endoscopes come in various sizes, but all have a tiny video camera at the tip of the endoscope. During an endoscopic procedure, this tiny video camera generates a video signal of the interior of a human organ, which is displayed on a monitor for real time analysis by the physician. Colonoscopy is an important screening tool for colorectal cancer. In the US, colorectal cancer is the second leading cause of all cancer deaths behind lung cancer [1]. As the name implies, colorectal cancers are malignant tumors that develop in the colon and rectum. The survival rate 
is higher if the cancer is found and treated early before metastasis to lymph nodes or other organs occurs.

The effectiveness of colonoscopy in prevention of colorectal cancers depends on the quality of the inspection of the colon, which generally can be evaluated in terms of the withdrawal time (time spent during the withdrawal phase) and the thoroughness of the inspection of the colon mucosa. Current American Society for Gastrointestinal Endoscopy (ASGE) guidelines suggest that (1) on average the withdrawal phase during a screening colonoscopy should last a minimum of 6 minutes and (2) the visualization of cecum anatomical landmarks such as the appendiceal orifice and the ileocecal valve should be documented [2].

Nevertheless, there was no automated measurement method to evaluate the endoscopist's skill and the quality of a colonoscopic procedure. To address this critical need, we have developed a prototype capturing system, which automatically records colonoscopic procedures on a hard disk in MPEG-2 format [3]. This system has been placed at Mayo Clinic Rochester since the beginning of February 2003 to capture colonoscopic procedures performed by de Groen (co-author) and colleagues.

The diagnostic accuracy of colonoscopy depends on the quality of the bowel preparation [4]. Inadequate cleansing can result in missed pathologic lesions. Colonic cleansing is mostly performed with solutions containing polyethylene glycol (PEG), and the alternatives are sodium phosphate, magnesium citrate, or bisacodyl [5]. The ideal preparation method would reliably empty the colon of all fecal material, and have little effect on the gross or the microscopic appearance of the mucosa. It would require a relatively short period for ingestion and evacuation, cause little patient discomfort, and produce no significant fluid-electrolyte shifts. It also should maximize the detection of colonic disease including polyps and carcinoma [5].

The quality of bowel cleansing is generally assessed by the quantity of solid or liquid stool in the lumen. Despite a large body of published data on methods that could optimize cleansing, a substantial level of inadequate cleansing occurs in $10 \%$ to $75 \%$ of patients in randomized controlled trials [6]. Poor bowel preparation has been associated with patient characteristics, such as inpatient status, history of constipation, use of antidepressants, and noncompliance with cleansing instructions. The American Society for Gastrointestinal Endoscopy (ASGE) and American College of Gastroenterology (ACG) Taskforce on Quality in Endoscopy suggested that every colonoscopy report should include an assessment of the quality of bowel preparation. They proposed the use of terms such as "excellent," "good," "fair," and "poor," but admitted that these terms lack standardized definitions [7]. To address this, the authors in [7] proposed the 'Boston Bowel Preparation Scale' (BBPS) in which the terms "excellent," "good," "fair," and "poor," were replaced by a four-point scoring system applied to each of the three broad regions of the colon: the right colon (including the cecum and ascending colon), the transverse colon (including the hepatic and splenic flexures), and the left colon (including the descending colon, sigmoid colon, and rectum). This scoring system will be discussed in Section 4 later.

This method is still based on some subjective evaluation of colon parts (i.e., just numbers instead of terms). An automatic method to identify stool in digitized images obtained during colonoscopy would obviate any subjective scoring methods and be a 
valuable asset among automated tools for measuring the quality of colonoscopy procedures.

A frame in colonoscopy video consists of a number of pixels as other digitized images typically do. Each pixel has three number values representing Red (R), Green $(\mathrm{G})$, and Blue (B), so each pixel can be plotted into 3-dimensional RGB color space. A set of stool pixels can form arbitrary shape(s) of volume(s) in 3-D RGB color space. If we can represent the volume(s) mathematically, we can decide automatically whether a pixel is a stool pixel or not. For the mathematical representation, we propose to use a set of planes (which will be discussed in more detail in Section 3). In this study, we propose two methods as follows:

- a method automatically deciding a percentage of stool area for each frame of colonoscopy video, and

- a method automatically computing an estimate of BBPS score based on the percentages of stool areas.

The rest of the paper is organized as follows. Section 2 discusses the background and related work. Section 3 and Section 4 describe the proposed methodology and the calculation of BBPS score, respectively. Section 5 shows our experimental results. Finally, Section 6 summarizes our concluding remarks.

\section{Background and Related Work}

Much research has focused on color based classification of images. In fact, color based classification plays a major role in the field of medical imaging. A huge number of articles based on color features has been published. The most common problem discussed within these articles deals with new positive class examples emerging after the training processes finish. We will briefly discuss two examples.

Zeki et al [8] proposed an incremental learning algorithm with SVM (Support Vector Machine) ensembles. The authors mainly focus on how to overcome a catastrophic misclassification problem of the SVM classifier by adding the ability to learn new instances. They propose to create a new ensemble of SVM classifier for the newly added data instances. The authors suggested to generate a number of classifiers for a given data set, and to keep a subset of most effective classifiers that are selected based on a weighted majority voting system. They combined the Learn++ algorithm with SVM to give it the ability to learn new instances. A major shortcoming of this approach is that the method is not applicable if there is not enough new data available to train a new SVM ensemble.

In our previous work [9], we proposed a method classifying stool images in colonoscopy videos using a SVM classifier. For each frame a vector is specified, and a color histogram is computed for each frame. The video frame is down-sampled into blocks in order to reduce the size of the feature vector. Features to the SVM classifier are, in fact, the mean value for each block. Then, the stool mask is applied to each video frame using the trained SVM classifier, and a post processing step is applied to improve the detection quality. The post processing step includes a majority filter and a binary area opening filter. Finally, frames having more than 5\% of stool 
area are classified as stool frames. It also has the catastrophic misclassification problem when it comes to learn new instances. And it lacks the ability to learn new data instances when available.

Our new method is preferable in detecting stool regions over the above mentioned methods because of the following;

- Our new method addresses the catastrophic misclassification problem (incremental learning) of SVM classifier. It can learn new instances instantly, and does not need a certain amount of data as in SVM classifier. Consequently, it is more accurate.

- Since our method can learn new instances instantly, its training process is fast. Also, its detecting process is fast because we can optimally reduce the number of comparisons.

\section{Classification and Detection Methodology}

The proposed method has the training and the detecting (or test) stages. The training stage has three steps: All stool pixel projection, Stool plane selection, and Stool plane modeling. As a result, the training stage generates a classification model which is used in the detecting stage. In this section, we will discuss the two stages.

\subsection{Training Stage}

Digital color images including our colonoscopy images are modeled in a RGB color space (cube) in which each color band is represented with 8-bit ranging from 0 to 255 , giving us a total of $256^{3}$ potential colors. Fig. 1 shows an example of frames that can be found in a colonoscopy video. We project all stool pixels in a frame into RGB color cube as the first step (All stool pixel projection). To discriminate stool pixels from non-stool pixels we use the fact that each color pixel has a unique location in the RGB color cube as three coordinates R, G, B as illustrated in Fig. 2(a). For convenience, $\mathrm{RGB}$ is mapped to the $\mathrm{XYZ}$ coordinate system as shown. In the second step (Stool plane selection), we put 256 planes into the RGB cube along the R (X) axis so that each integer location on the $R$ axis has a plane parallel to a GB plane as seen in Fig. 2 (b). It is possible to put planes along the G or B axis - doing so will not alter the classification modeling. In our study we selected the $\mathrm{R}$ axis along which we put planes. We assign a number (from 0 to 255 ) to each plane (i.e., Plane\#0, Plane\#1, ... Plane\#255). One assigned number to each plane is sufficient since all planes are perpendicular to the GB (YZ) plane. Among these 256 planes, we select only planes with stool pixels. Each selected plane is called a 'Positive Plane'.

Each positive plane contains a projection of stool pixels at the corresponding location, and is treated as a 2D classifier at the relevant location. For instance, Plane\#0 at the location $(0,0,0)$ is treated as a classifier for positive class examples (stool pixels in our case) that has a R (X) value of zero (0). This method inherits fast classification as it already possesses the property of eliminating non-relevant class examples (i.e., non-stool pixels in our case) in the training process. 


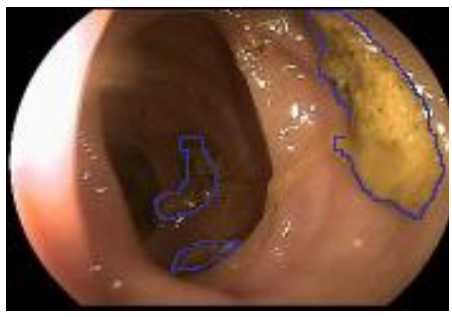

(a)

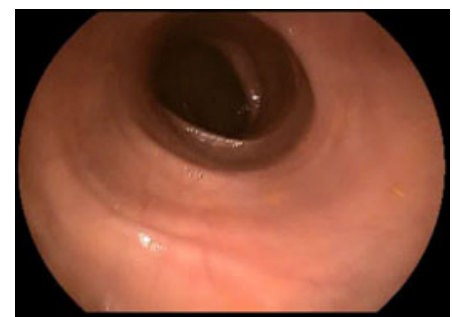

(b)

Fig. 1. Examples of (a) Stool- marked with blue (b) Non-stool Frames

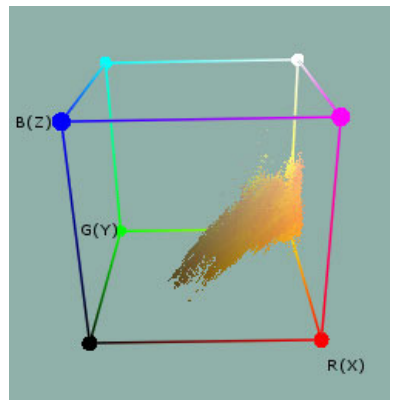

(a)

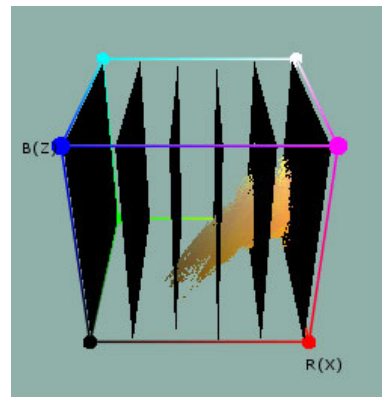

(b)

Fig. 2. (a) RGB cube and corresponding locations of stool pixels, and mapping of RGB axis to XYZ axis (within brackets), and (b) Several planes inserted into the RGB cube of (a)

In the third step (Stool plane modeling), we model the areas of positive class examples (stool pixels). First, a positive plane (Fig. 3(a)) is divided into four blocks. A block is a square since each plane is a square (256 x 256), and each may contain all stool pixels, all non-stool pixels, or mixture of non-stool and stool pixels. For all four blocks, we check the following three conditions. If all (or more than 95\%) of pixels in a block are positive class examples (stool pixels), the block becomes a positive block, and the procedure for this block is done. If all pixels in a block are non-positive class examples (non-stool pixels), then the block becomes a negative block, and the procedure for this block is done. If some (less than 95\%) of pixels in a block are positive class examples (stool pixels), and the block has more than or equal to the MNP (Minimum Number of Pixels - MNP is 16 in our case), the block is divided into four smaller blocks, and we check the above three conditions for all four smaller blocks. The minimum block size is $4 \mathrm{x} 4$. When the iteration reaches the minimum block size, a block becomes a positive block if it has more positive class examples (stool pixels). Otherwise, it becomes negative block. This procedure is recursive, and the blocks become smaller in the next iteration (Each block is divided by four at each iteration). In case the block has less than the MNP and it has more positive class examples (stool pixels), then it becomes a positive block. Otherwise, it becomes a negative block. All levels of blocks have their own unique number values in the way shown in Fig. 3(c). It is a very convenient and non-ambiguous way for 
numbering. Among these numbers, a set of numbers for positive blocks can form a vector for a positive plane, and a set of vectors from all positive planes can form a classification model for the detecting stage. This model possesses an incremental learning property. Its incremental learning is performed as follows. When there is a new positive pixel to be inserted into the model, we can find a corresponding minimum size ( $4 \times 4)$ block which is a negative block. The block can become a positive block if it gets more positive class examples (stool pixels) by adding this new positive pixel. In this way, we do not have to run the entire training process from the beginning when we need to add additional positive examples.

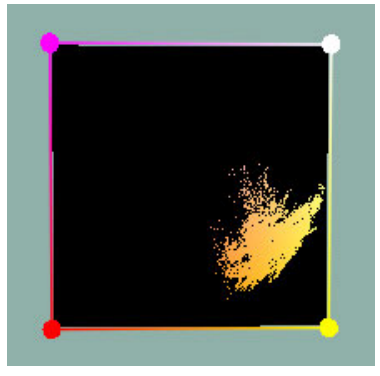

(a)

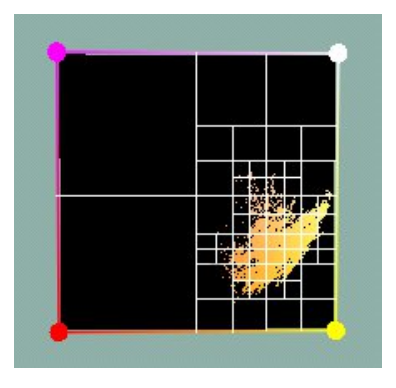

(b)

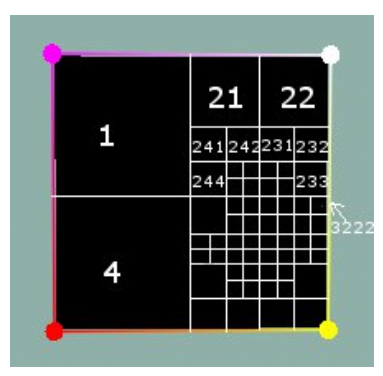

(c)

Fig. 3. (a) Positive class examples (stool pixels) projected on a positive plane (plane\#250) as looking into the RGB cube from right side in Fig. 2(b), (b) Minimum coverage area of the positive classes examples (stool pixels), and (c) Unique numbering of blocks for fast access (not all shown for clarity) - clockwise numbering starting from top left quadrant

\subsection{Detecting Stage}

Detection of the stool pixel is performed by evaluating a candidate pixel on the classification model generated in Section 3.1. Once there is pixel to be detected, the $\mathrm{R}$ (X) value of the pixel is obtained and used as the index to select the corresponding positive plane. For example, if the $\mathrm{R}(\mathrm{X})$ value is 5, then Plane\#5 is selected and examined. This will dramatically reduce the number of comparisons so that the analysis time is significantly reduced. In other words, the analysis time to determine pixel stool class of the proposed technique is not dependent on the number of positive planes, but on how many positive blocks there are in the corresponding plane, which is usually very small. By comparing the GB (YZ) values of the pixel with the vector obtained from the third step of the training stage (discussed in Section 3.1), we can determine whether it can be classified as a positive class (stool) pixel. Otherwise, it is classified as negative (non-stool) class pixel. After all pixels of a frame are evaluated, we can calculate a percentage of stool area for each frame: the number of all stool pixels divided by the number of total pixels.

\section{Computing BBPS Score}

In this section, we will discuss a method automatically compute an estimate of the BBPS score based on the percentages of stool areas obtained in Section 3. As 
mentioned in Section 1 (Introduction), the authors in [7] proposed 'Boston Bowel Preparation Scale' (BBPS) in which the terms "excellent," "good," "fair," and "poor," were replaced by a four-point scoring system applied to each of the three broad regions of the colon: the right colon (including the cecum and ascending colon), the transverse colon (including the hepatic and splenic flexures), and the left colon (including the descending colon, sigmoid colon, and rectum). These six parts of colon can be seen in Fig. 4, and the relationships between the terms and the points can also be seen in Table 1 .

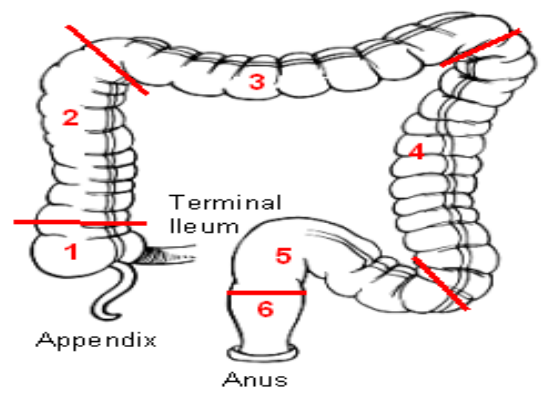

Fig. 4. Six parts of Colon: 1 - Cecum, 2 - Ascending colon, 3 - Transverse colon, 4 Descending colon, 5 - Sigmoid, and 6 - Rectum

Table 1. Relationship between the quality terms and the quality points

\begin{tabular}{cc}
\hline Quality Term & Quality Point \\
\hline Excellent & 3 \\
Good & 2 \\
Fair & 1 \\
poor & 0 \\
\hline
\end{tabular}

The points in Table 1 are assigned as follows:

- $0=$ Unprepared colon segment with mucosa not seen due to solid stool that cannot be cleared.

- 1 = Portion of mucosa of the colon segment seen, but other areas of the colon segment not well seen due to staining, residual stool and/or opaque liquid.

- 2 = Minor amount of residual staining, small fragments of stool and/or opaque liquid, but mucosa of colon segment seen well.

- $\quad 3$ = Entire mucosa of colon segment seen well with no residual staining, small fragments of stool or opaque liquid.

Each region of the colon receives a "segment score" from 0 to 3 and these segment scores are summed for a total BBPS score ranging from 0 to 9 . Therefore, the maximum BBPS score for a perfectly clean colon without any residual liquid is 9 , and the minimum BBPS score for an unprepared colon is 0 . 
We compute an estimate of the BBPS score automatically for a recorded colonoscopy video. A colonoscopy video consists of two phases: an insertion phase and a withdrawal phase as seen in Figure 5. During the insertion phase, a flexible endoscope (a flexible tube with a tiny video camera at the tip) is advanced under direct vision via the anus into the rectum and then gradually into the cecum (the most proximal part of the colon) or the terminal ileum. During the withdrawal phase, the endoscope is gradually withdrawn. The purpose of the insertion phase is to reach the cecum or the terminal ileum. Careful mucosa inspection and diagnostic or therapeutic interventions such as biopsy, polyp removal, etc., are performed during the withdrawal phase.

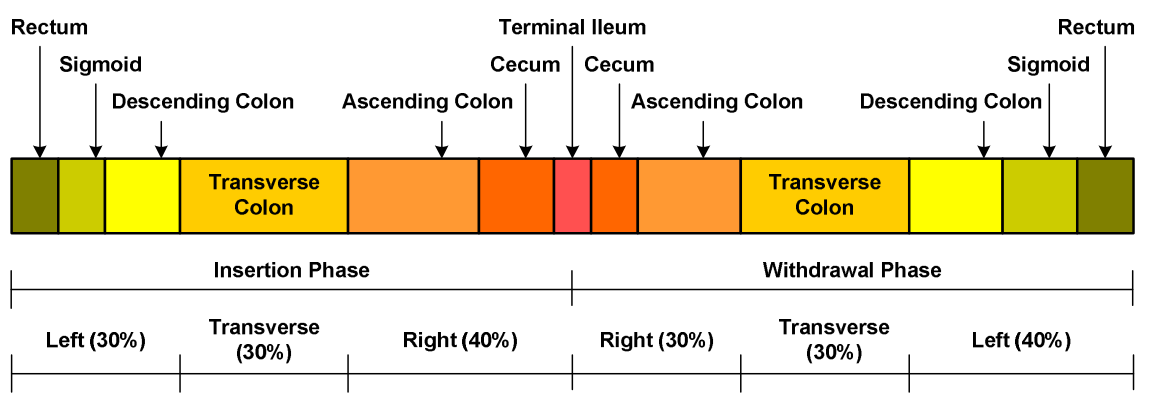

Fig. 5. Two Phases in Colonoscopy Video

The recorded colonoscopy video is divided into insertion phase and withdrawal phase automatically using the techniques we developed [10]. In our estimate of BBPS implementation, the right colon has the last $40 \%$ of insertion phase plus the first $30 \%$ of withdrawal phase. The transverse colon has the middle $30 \%$ of insertion phase plus the middle $30 \%$ of withdrawal phase. The left colon has the first $30 \%$ of insertion phase plus the last $40 \%$ of withdrawal phase. These numbers are based on experiments and opinion of the domain expert. We calculate estimated BBPS score values mathematically based on the stool percentage values obtained above for each frame. We assign a score value for each frame based on the stool pixel percentage present in the frame, and calculate the numerical average for each colon segment (right colon, transverse colon, and left colon) for the final score value. The stool percentage values and the corresponding score values are estimates based on the original images of the BBPS description and are shown in the table 2.

Table 2. Stool percentage in a frame and the assigned score value

\begin{tabular}{cc}
\hline Stool percentage \% & Score value assigned \\
\hline $0-10$ & 3 \\
$11-25$ & 2 \\
$26-50$ & 1 \\
$51-100$ & 0 \\
\hline
\end{tabular}




\section{Experimental Results}

All the computations in our experiments were performed on a PC-compatible workstation with an Intel Pentium D CPU, 1GB RAM, and Windows XP operating system. For our experiments, we used 58 videos recorded with Fujinon colonoscopes. The average length of the videos is around 20 minutes, and their frame size is $720 \mathrm{x}$ 480 pixels. This section is divided into two subsections; one for assessing the proposed stool detection and the other for BBPS score calculation.

\subsection{Stool Detection}

We randomly extracted 1,000 frames from all 58 videos, in which each frame has at least one stool region. The domain experts marked and confirmed the positive (stool) regions in these frames. From half (500) of these frames, we filtered out duplicate examples (pixels), and obtained only unique positive examples (stool pixels) for the training. Table 3 shows the stool and non-stool pixels used for training. Using 31,858 stool pixels, we followed all the steps in Section 3.1. Then, we used all the pixels in the remaining half (500) of the frames for the detecting stage discussed in Section 3.2. We assess the effectiveness of our proposed algorithm at the pixel level by determining the performance metrics Sensitivity and Specificity. For a comparison purpose, we implemented the method in our previous work [9] using the same dataset mentioned above (also seen in Table 3). Table 4 shows this comparison. As seen in the table, the proposed method is better than the previous one in terms of sensitivity and specificity.

Table 3. Number of examples (pixels) used in the training stage

\begin{tabular}{ll}
\hline & Stool Dataset \\
\hline $\begin{array}{l}\text { Positive } \\
\text { (stool) }\end{array}$ & 31,858 \\
$\begin{array}{l}\text { Negative } \\
\text { (non-stool) }\end{array}$ & 52,434 \\
\hline
\end{tabular}

Table 4. Performance comparison with previous work

\begin{tabular}{lccc}
\hline \multicolumn{2}{c}{ Sensitivity } & \multicolumn{2}{c}{ Specificity } \\
\hline New & Old & New & Old \\
\hline $92.9(\%)$ & 90.6 & 95.0 & 93.8 \\
\hline
\end{tabular}

Also, we implemented the well-known KNN (K-Nearest Neighbor, K=1 in our study) classifier using the same dataset mentioned above to see how fast the proposed method can perform. Table 5 presents the speed comparison for KNN classifier with our proposed method. It takes more than 420 seconds ( 7 minutes) to evaluate a frame (720 x 480 pixels) in the KNN. On average, it takes 0.00127 seconds to evaluate one pixel. However, it takes around 11 seconds to evaluate a frame (720 x 480 pixels) 
in the proposed method. If we consider only the detection stage, it takes less than one second to evaluate one frame. This is a significant achievement. We need to process 3,600 frames to generate a colonoscopy report for a 20 minute colonoscopy video if we analyze 3 frames per second $(3$ frames/second $* 60$ seconds/minute $* 20$ minutes $=$ 3600 frames). Thus, it is not practical to use KNN classifier even though it can provide $98 \%$ of sensitivity and specificity on average.

Table 5. Average Time taken for KNN and the proposed method

\begin{tabular}{ccc}
\hline KNN (trainging +detection) & $\begin{array}{c}\text { Proposed method } \\
\text { (Detection) }\end{array}$ & $\begin{array}{c}\text { Prposed Method } \\
\text { (Training ) }\end{array}$ \\
\hline 437.5 (seconds) & 0.9 & 10.0 \\
\hline
\end{tabular}

Fig. 6 lists some results obtained using the proposed method. The numbers (1, 2 and 3) on each frame represent the regions semi-automatically segmented for the determination of ground truth. For instance, region 2 in Fig. 6(a), region 1 in Fig. 6(b), and region 1 in Fig. 6(c) were labeled as stool by the domain experts. The first row consists of the original frames with the ground truth marked, and the second row contains the results from our method for the first row (stool regions are marked with blue).

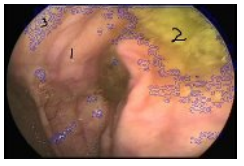

(a)

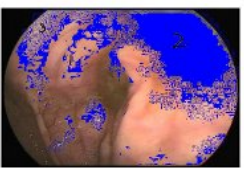

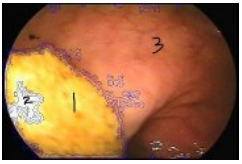

(b)

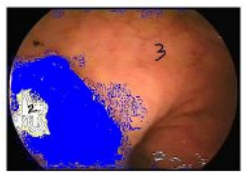

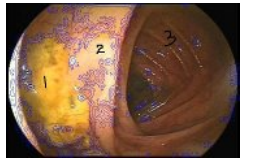

(c)

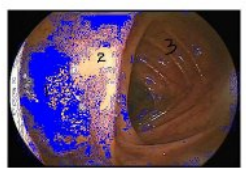

Fig. 6. Sample results for stool detection

\subsection{BBPS Calculation}

We generated estimates of BBPS scores for all 58 videos and list randomly selected 5 results in Table 6 having a comparison with the ground truth scores suggested by domain experts. The column 'Ground truth BBPS' in Table 6 is the average score values from three different experts. It is rare to find video files where all three experts agree this close about the scores. Therefore, it is hard to find a definitive Ground Truth score for a given video. We took the average of three BBPS ground truths as our target value to be reached. As seen in the table, the calculated values are very close to the ground truths. 
Table 6. Comparision of Calculated BBPS scores with Ground Truth BBPS scores

\begin{tabular}{ccc}
\hline Video ID & Calculated BBPS & Ground Truth BBPS \\
\hline 1.mpg & 7 & 6 \\
$5 . \mathrm{mpg}$ & 4 & 3 \\
$9 . \mathrm{mpg}$ & 6 & 6 \\
$10 . \mathrm{mpg}$ & 6 & 7 \\
$13 . \mathrm{mpg}$ & 6 & 5 \\
\hline
\end{tabular}

\section{Conclusion and Future Work}

The two most critical aspects of colonoscopy that determine its protective effect against development of colorectal cancer are the quality of bowel cleansing and technical performance of the endoscopist. Neither of these two aspects can be objectively measured in a manual fashion. Recent reports of "missed" polyps and cancers suggest that the protective effect of colonoscopy is far from complete, raise questions about the quality of bowel cleansing as well as technical performance, and call for new methods to measure and improve the quality of colonoscopy. Indeed, automated video analysis techniques have recently been introduced to objectively determine technical performance. In this paper, we present an automated method to detect stool regions based on the color features using new classification modeling. Our preliminary investigation shows that our stool detection method is able to detect stool with very high accuracy achieving sensitivity over $93 \%$ with $95 \%$ specificity. Our previous work for stool detection [9] was very good, but had its limitations. First, the training data were images derived from a single patient; but in this new study, we used 58 different videos from 58 different patients. Second, a global, objective "colon cleansing" score was needed to be developed representing a composite of all individual image scores and tested against one or more manual "colon cleansing" scores. In this new study, we implemented a method to compute an estimate of the BBPS score automatically, and compared the score with the ground truths provided by domain experts. Our new method shows improved performance and can be applied in colonoscopy practice for quality measurements. In addition, our method has the ability to learn new positive class examples without running the entire training process from beginning as we can adjust each plane separately. This adds to our method the valuable ability of incremental learning. However, further research is necessary. For example, stool varies in consistency from solid lumps to transparent water-diluted fluid; our training data consisted of images where the outline of solid or non-transparent, liquid stool was marked as "stool". Thus mucosa with just a thin cover of semi-transparent liquid stool was not included in our training data. This means that not the entire amount of stool was targeted for recognition. Clearly, more testing is needed to determine how well our algorithms hold up under variable, reallife circumstances. 
Acknowledgement. This work is partially supported by NSF STTR-Grant No. 0740596, 0956847, National Institute of Diabetes and Digestive and Kidney Diseases (NIDDK DK083745), and the Mayo Clinic. Any opinions, findings, conclusions, or recommendations expressed in this paper are those of authors. They do not necessarily reflect the views of the funding agencies. Johnny Wong, Wallapak Tavanapong, and JungHwan Oh hold positions at EndoMetric Corporation, Ames, IA 50014, U.S.A, a for profit company that markets endoscopy-related software. Johnny Wong, Wallapak Tavanapong, JungHwan Oh, and Mayo Clinic own stocks in EndoMetric. Piet de Groen, Johnny Wong, Wallapak Tavanapong, JungHwan Oh and Mayo Clinic have received royalty payments from EndoMetric.

\section{References}

1. American Cancer Society.:Colorectal Cancer Facts and Figures, American Cancer Society Special Edition 2005, pp. 1-20 (2005)

2. Douglas, K.R., John, L.P., Todd, H.B., Amitabh, C., Jonathan, C., Stephen, E.D., Brenda, H., Brian, C.J., Klaus, M., Bret, T.P., Michael, A.S., Douglas, O.F., Irving, M.P.: Quality Indicators for Colonoscopy. American Journal of Gastroenterology 101, 873-885 (2006)

3. Stanek, S., Tavanapong, W., Wong, J., Oh, J., de Groen, P.C.: Automatic Real-Time Capture and Segmentation of Endoscopy Video. PACS and Imaging Informatics. In: SPIE Medical Imaging, vol. 6919, pp. 69190X-69190X-10 (February 2008)

4. Cappel, M.S., Friedel, D.: The Role of Sigmoidoscopy and Colonoscopy in the Diagnosis and Management of Lower Gastrointestinal Disorders: Endoscopic Findings, Therapy, and Complications. Medical Clinics of North America 86, 1253-1288 (2002)

5. Ernstoff, J.J., Howard, D.A., Marshall, J.B., Jumshyd, A., Mc-Cullough, A.J.: A Randomised Blinded Clinical Trial of a Rapid Colonic Lavage Solution (Golytely) Compared with a Standard Preparation for Colonoscopy and Barium Enema. Gastroenterology 84, 1512-1516 (1983)

6. Ness, R.M., Manam, R., Hoen, H.J., Chalasani, N.: Predictors of Inadequate Bowel Preparation for Colonoscopy. American Journal of Gastroenterology 96, 1797-1802 (2001)

7. Lai, E.J., Calderwood, A.H., Doros, G., et al.: The Boston Bowel Preparation Scale: a valid and reliable instrument for colonoscopy-oriented research. Gastrointestinal Endoscopy Clinics of North America 69, 620-625 (2009)

8. Erdem, Z., Polikar, R., Gurgen, F., Yumusak, N.: Ensemble of SVMs for Incremental Learning. In: Oza, N.C., Polikar, R., Kittler, J., Roli, F. (eds.) MCS 2005. LNCS, vol. 3541, pp. 246-256. Springer, Heidelberg (2005), doi:10.1007/11494683_25

9. Hwang, S., Oh, J., Tavanapong, W., Wong, J., de Groen, P.C.: Stool Detection in Colonoscopy Videos. In: Proc. of International Conference of the IEEE Engineering in Medicine and Biology Society (EMBC), Vancouver, British Columbia, Canada, pp. 30043007 (August 2008)

10. Oh, J., Rajbal, M.A., Muthukudage, J.K., Tavanapong, W., Wong, J., de Groen, P.C.: Real-Time Phase Boundary Detection in Colonoscopy Videos. In: Proc. of 6th International Symposium on Image and Signal Processing and Analysis (ISPA 2009), Salzburg, Austria, September 16-18, pp. 724-729 (2009) 